\title{
Cooperative MIMO Channel Modeling and Multi-Link Spatial Correlation Properties
}

\author{
Xiang Cheng, Member, IEEE, Cheng-Xiang Wang, Senior Member, IEEE, Haiming Wang, Xiqi Gao, Senior \\ Member, IEEE, Xiao-Hu You, Senior Member, IEEE, Dongfeng Yuan, Senior Member, IEEE, Bo Ai, Senior \\ Member, IEEE, Qiang Huo, Ling-Yang Song, Member, IEEE, and Bing-Li Jiao Member, IEEE
}

\begin{abstract}
In this paper, a novel unified channel model framework is proposed for cooperative multiple-input multiple-output (MIMO) wireless channels. The proposed model framework is generic and adaptable to multiple cooperative MIMO scenarios by simply adjusting key model parameters. Based on the proposed model framework and using a typical cooperative MIMO communication environment as an example, we derive a novel geometry-based stochastic model (GBSM) applicable to multiple wireless propagation scenarios. The proposed GBSM is the first cooperative MIMO channel model that has the ability to investigate the impact of the local scattering density (LSD) on channel characteristics. From the derived GBSM, the corresponding multi-link spatial correlation functions are derived and numerically analyzed in detail.
\end{abstract}

Index Terms-Cooperative MIMO channels, geometry-based stochastic model, spatial correlation, non-isotropic scattering.

Manuscript received 15 February 2011; revised 20 July 2011. The authors would like to acknowledge the support from the RCUK for the UK-China Science Bridges Project: R\&D on (B) $4 G$ Wireless Mobile Communications. The work of $\mathrm{X}$. Cheng was jointly supported by the National Natural Science Foundation of China (Grant no. 61101079), the Science Foundation for the Youth Scholar of Ministry of Education of China (Grant no. 20110001120129), the State Key Laboratory of Rail Traffic Control and Safety (RCS 2010K009), Beijing Jiaotong University, the Key Laboratory of Wireless Sensor Network \& Communication (Grant no. 2011004), Shanghai Institute of Microsystem and Information Technology, Chinese Academy of Sciences, Important National Science \& Technology Specific Project of China (Grant no. 2010ZX03001-002), Major Program of National Natural Science Foundation of China (60830001), Program for Changjiang Scholars and Innovative Research Team in University (IRT 0949), Program for New Century Excellent Talents in University (NCET-09-0206), and State Key Laboratory of Rail Traffic Control and Safety (RCS 2008ZZ006), Beijing Jiaotong University. C.$\mathrm{X}$. Wang would also like to acknowledge the support by the Scottish Funding Council for the Joint Research Institute in Signal and Image Processing with the University of Edinburgh, as part of the Edinburgh Research Partnership in Engineering and Mathematics (ERPem), and by the Opening Project of Key Laboratory of Cognitive Radio and Information Processing (Guilin University of Electronic Technology), Ministry of Education (Grant No.: 2011KF01). The work of H.-M. Wang was supported in part by the National Science and Technology Major Project of China under Grant 2009ZX03007-003-02.

X. Cheng, Q. Huo, S.-L. Yang, and B.-L. Jiao are with the School of Electronics and Computer Science, Peking University, Beijing 100871, China (e-mail: $\{$ xiangcheng, qiang.huo, lingyang.song, jiaobl $\} @$ pku.edu.cn). $\mathrm{X}$. Cheng is also with the State Key Laboratory of Rail Traffic Control and Safety, Beijing Jiaotong University, and Key Laboratory of Wireless Sensor Network \& Communication, Shanghai Institute of Microsystem and Information Technology, Chinese Academy of Sciences.

C.-X. Wang is with the Joint Research Institute for Signal and Image Processing, School of Engineering \& Physical Sciences, Heriot-Watt University, Edinburgh EH14 4AS, UK (e-mail: cheng-xiang.wang@hw.ac.uk). (corresponding author)

H. Wang, X. Gao, and X.-H. You are with the School of Information Science and Engineering, Southeast University, Najing, 210096, China (email: \{hmwang, xqgao, xhyu $\} @$ seu.edu.cn).

D. Yuan is with the School of Information Science and Engineering, Shandong University, Jinan, 250100, China (e-mail: dfyuan@sdu.edu.cn).

B. Ai is with the State Key Laboratory of Rail Traffic Control and Safety, Beijing Jiaotong University, Beijing 100044, China (e-mail: boai@bjtu.edu.cn).

Digital Object Identifier 10.1109/JSAC.2012.120218.

\section{INTRODUCTION}

$\mathbf{C}$ ONVENTIONAL multiple-input multiple-output (MIMO) technology, known as point-to-point MIMO, has been widely used in many standards [1]- [3] due to its ability to significantly enhance the performance of wireless communication systems [4]- [5]. Research on cooperative MIMO technologies has recently received much attention [6]- [8]. Cooperative MIMO, also known as virtual MIMO or distributed MIMO, groups multiple radio devices to form virtual antenna arrays so that they can cooperate with each other by exploiting the spatial domain of mobile fading channels. As a new emerging technology, many research challenges in cooperative communications have to be addressed before the wide deployment. Detailed knowledge about the underlying propagation channels and the corresponding channel models are the fundamental to meet those challenges for the better design of cooperative MIMO systems [9].

Several papers have reported measurements of various statistical properties of cooperative MIMO channels for different scenarios. A few indoor cooperative channel measurements were reported in [10], where the cooperative nodes are all static. Mobile multi-link measurements were presented in [11] for indoor cooperative MIMO channels. Outdoor cooperative MIMO channel measurements were addressed in [12] and [13] for static nodes and mobile nodes, respectively. All these measurement campaigns concentrated on the investigation of the channel characteristics of individual links for different scenarios, such as path loss, shadow fading, and small scale fading. Unlike conventional point-to-point MIMO systems, cooperative MIMO systems consist of multiple radio links that may exhibit strong correlations, e.g., base station (BS)BS, BS-relay station (RS), RS-RS, RS-mobile station (MS), BS-MS, and MS-MS links. The correlation of multiple links exists due to the environment similarity arising from common shadowing objects and scatterers contributing to different links and can significantly affect the performance of cooperative MIMO systems. The investigation of the correlations between different links is rare in the current literature.

The multi-link correlation consists of large scale fading correlation and small scale fading correlation. Only a few papers have analyzed and modeled large scale fading correlations, including shadow fading correlation, delay spread correlation, and azimuth correlation. The 3rd Generation Partnership Project (3GPP) Spatial Channel Model (SCM) [14], the Wireless World Initiative New Radio Phase II (WINNER II) channel model [15], and the IEEE 802.16j 
channel model [16] all investigated and modeled large scale fading correlations of different links for multiple scenarios. However, as mentioned in [17], these correlation models are not consistent and a unified correlation model for large scale fading is necessary. Recently, in [18] a unified framework that can investigate both static and dynamic shadow fading correlations was proposed for indoor and outdoor-to-indoor scenarios. There are even fewer papers available investigating small scale fading correlations. In [19], the authors proposed a multiuser MIMO channel model focusing on the investigation of the impact of surface roughness on spatial correlations. In [17], a preliminary investigation on spatial correlations for coordinated multi-point (CoMP) transmissions was reported. The investigation on spatial correlations of multilink propagation channels in amplify-and-forward (AF) relay systems was reported in [20]. However, all the aforementioned investigations on multi-link spatial correlations are scenariospecific. For example, [19] only modeled the scenario where scatterers are located in streets, [17] only focused the CoMP scenario, and [20] only investigated the AF relay scenario. A unified channel model framework to investigate multilink small scale fading correlations for different scenarios is therefore highly desirable.

To fill the aforementioned gap, this paper proposes a unified channel model framework for cooperative MIMO systems and investigates spatial correlations of different links in multiple scenarios. The main contributions and novelties of this paper are listed as follows.

1) We propose a wideband unified channel model framework that is suitable to mimic different links in cooperative MIMO systems, such as the BS-BS/RS/MS link, RS-RS/MS link, and MS-MS link. Due to different local scattering environments around BSs, RSs, and MSs, a high degree of link heterogeneity or variations is expected in cooperative MIMO systems. In this paper, we are interested in various cooperative MIMO environments which can be classified based on the physical scenarios and application scenarios. The physical scenarios include outdoor macro-cell, micro-cell, pico-cell, and indoor scenarios. Each physical scenario further includes 3 application scenarios, i.e., BS cooperation, MS cooperation, and relay cooperation. Therefore, 12 cooperative MIMO scenarios are considered in this paper and the proposed framework can be adapted to the 12 scenarios by simply adjusting key model parameters.

2) Taking a cooperative relay system, which includes three links (BS-RS, RS-MS, and BS-MS), as an example, we show how to apply the proposed channel model framework and derive a novel geometry-based stochastic model (GBSM) for multiple physical scenarios. The proposed GBSM is the first cooperative MIMO channel model that has the ability to mimic the impact of the local scattering density (LSD) on channel characteristics.

3) From the proposed GBSMs and taking the spatial correlation between the BS-RS link and the BS-MS link as an example, we further derive the multi-link spatial correlation functions.

\section{A Unified Cooperative Mimo Channel Model FRAMEWORK}

Cooperative MIMO channel measurements [18], [21] have clearly demonstrated that the degree of link heterogeneity in cooperative MIMO systems is highly related to local scattering environments around different devices. Therefore, the cooperative MIMO model framework needs to reflect the influence of different local scattering environments on the link heterogeneity for different scenarios while keeping the acceptable model complexity.

Let us now consider a general wideband cooperative MIMO system where all nodes are surrounded by local scatterers and a link between Node $A$ and Node $B$ is presented as shown in Fig. 1. It is assumed that each node can be in motion and is equipped with $L$ antenna elements. The proposed unified channel model framework expresses the channel impulse response (CIR) between the $p$ th antenna in Node $A$ and the $q$ th antenna in Node $B$ as the superposition of line-of-sight (LoS) and scattered rays

$$
h_{p q}(t, \tau)=h_{p q}^{L o S}(t, \tau)+\sum_{i=1}^{I} \sum_{g=1}^{f_{I}(i)} h_{p q}^{i g}(t, \tau)
$$

where $I \geq 1$ is the number of related local scattering areas, $f_{I}(i)=\frac{I !}{(I-i) ! \cdot i !}$ denotes the total number of $i$-bounced components, and $h_{p q}^{i g}(t, \tau)$ represents the $g$ th scattered component consisting of $i$-bounced rays. For example, $h_{p q}^{21}(t, \tau)$ denotes the first double-bounced component. It is worth noting that the parameter $f_{I}(i)$ is obtained not purely based on the number of related local scattering areas, but also according to the following practical criterion: the $i$-bounced waves are always bounced by $i$ scatterers located in different local scattering areas from far to near relative to the receiver. Based on this practical criterion, some $i$-bounced components are not necessarily to be considered, which makes the proposed model more practical. For the cooperative communication environment shown in Fig. 1 with $I=4$, the proposed model framework consists of the LoS component, $f_{4}(1)=4$ singlebounced components, $f_{4}(2)=6$ double-bounced components, $f_{4}(3)=4$ triple-bounced components, and $f_{4}(4)=1$ quadruplebounced component. While other multi-bounced components that violate the aforementioned practical criterion are not included in the proposed model framework, such as the $A_{p}-S_{B}-S_{C}-B_{q}$ double-bounced component.

In the proposed model framework (1), the LoS component of the CIR is deterministic and can be expressed as [22]

$$
\begin{aligned}
h_{p q}^{L o S}(t, \tau) & =\sqrt{\frac{K_{p q} \Omega_{p q}}{K_{p q}+1}} e^{-j 2 \pi \lambda^{-1} \chi_{p q}} e^{j 2 \pi f_{m a x}^{A} t \cos \left(\alpha_{p q}^{L o S}-\gamma_{A}\right)} \\
& \times e^{j 2 \pi f_{\text {max }}^{B} t \cos \left(\phi_{p q}^{L o S}-\gamma_{B}\right)} \delta\left(\tau-\tau_{L o S}\right)
\end{aligned}
$$

where $\chi_{p q}$ is the travel path of the LoS waves through the link between $A_{p}$ and $B_{q}\left(A_{p}-B_{q}\right.$ link $), \tau_{L o S}$ denotes the LoS time delay, and $\lambda$ is the wave length with $\lambda=c / f$ where $c$ is the speed of light and $f$ is the carrier frequency. The symbols $K_{p q}$ and $\Omega_{p q}$ designate the Ricean factor and the total power of the $A_{p}-B_{q}$ link, respectively. Parameters $f_{\max }^{A}$ and $f_{\max }^{B}$ 


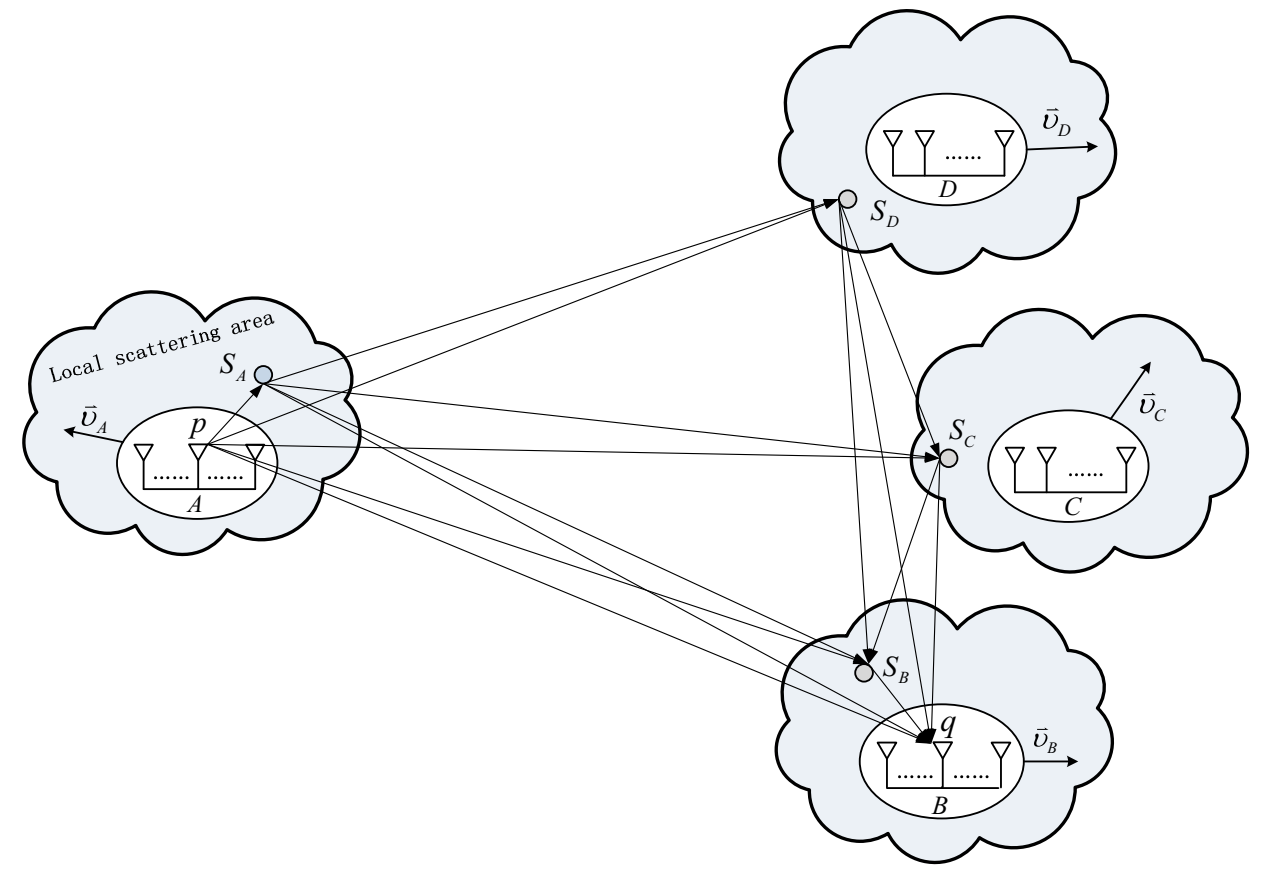

\begin{tabular}{|c|c|}
\hline LoS Component & $A_{p} \rightarrow B_{q}: h_{p q}^{L O S}(t, \tau)$ \\
\hline $\begin{array}{l}i=1, \quad \text { Single-bounced } \\
\text { Components }\end{array}$ & $\begin{array}{ll}A_{p} \rightarrow S_{B} \rightarrow B_{q}: h_{p q}^{11}(t, \tau) & A_{p} \rightarrow S_{C} \rightarrow B_{q}: h_{p q}^{12}(t, \tau) \\
A_{p} \rightarrow S_{D} \rightarrow B_{q}: h_{p q}^{13}(t, \tau) & A_{p} \rightarrow S_{A} \rightarrow B_{q}: h_{p q}^{14}(t, \tau)\end{array}$ \\
\hline $\begin{array}{l}i=2 \text {, Double-bounced } \\
\text { Components }\end{array}$ & $\begin{array}{ll}A_{p} \rightarrow S_{A} \rightarrow S_{B} \rightarrow B_{q}: h_{p q}^{21}(t, \tau) & A_{p} \rightarrow S_{A} \rightarrow S_{C} \rightarrow B_{q}: h_{p q}^{22}(t, \tau) \\
A_{p} \rightarrow S_{A} \rightarrow S_{D} \rightarrow B_{q}: h_{p q}^{23}(t, \tau) & A_{p} \rightarrow S_{D} \rightarrow S_{B} \rightarrow B_{q}: h_{p q}^{24}(t, \tau) \\
A_{p} \rightarrow S_{D} \rightarrow S_{C} \rightarrow B_{q}: h_{p q}^{25}(t, \tau) & A_{p} \rightarrow S_{C} \rightarrow S_{B} \rightarrow B_{q}: h_{p q}^{26}(t, \tau)\end{array}$ \\
\hline $\begin{array}{l}i=3, \quad \text { Triple-bounced } \\
\text { Components }\end{array}$ & $\begin{array}{ll}A_{p} \rightarrow S_{A} \rightarrow S_{D} \rightarrow S_{B} \rightarrow B_{q}: h_{p q}^{31}(t, \tau) & A_{p} \rightarrow S_{A} \rightarrow S_{D} \rightarrow S_{C} \rightarrow B_{q}: h_{p q}^{32}(t, \tau) \\
A_{p} \rightarrow S_{A} \rightarrow S_{C} \rightarrow S_{B} \rightarrow B_{q}: h_{p q}^{33}(t, \tau) & A_{p} \rightarrow S_{D} \rightarrow S_{C} \rightarrow S_{B} \rightarrow B_{q}: h_{p q}^{34}(t, \tau)\end{array}$ \\
\hline $\begin{array}{l}i=4, \\
\text { Quadruple-bounced } \\
\text { Components }\end{array}$ & $A_{p} \rightarrow S_{A} \rightarrow S_{D} \rightarrow S_{C} \rightarrow S_{B} \rightarrow B_{q}: h_{p q}^{41}(t, \tau)$ \\
\hline
\end{tabular}

Fig. 1. Geometry of a unified cooperative MIMO channel model framework.

are the maximum Doppler frequency with respect to Node $A$ and Node $B$, respectively, $\gamma_{A}$ and $\gamma_{B}$ are the angles of motion with respect to Node $A$ and Node $B$, respectively, and $\alpha_{p q}^{L o S}$ and $\phi_{p q}^{L o S}$ denote the angles of arrival/departure of the LoS path with respect to Node $A$ and Node $B$, respectively. The scattered component of the CIR in (1) can be shown as [22]

$$
\begin{aligned}
h_{p q}^{i g}(t, \tau) & =\sqrt{\frac{\eta_{p q}^{i g} \Omega_{p q}}{K_{p q}+1}} \lim _{\left\{N_{k}^{g}\right\}_{k=1}^{i} \rightarrow \infty} \sum_{\left\{n_{k}^{g}\right\}_{k=1}^{i}=1}^{\left\{N_{k}^{g}\right\}_{k=1}^{i}} \frac{1}{\sqrt{\prod_{k=1}^{i} N_{k}^{g}}} \\
& \times e^{j\left(\psi_{\left\{n_{k}^{g}\right\}_{k=1}^{i}}-2 \pi \lambda^{-1} \chi_{p q,\left\{n_{k}^{g}\right\}_{k=1}^{i}}\right)} \\
& \times e^{j 2 \pi f_{\text {max }}^{A} t \cos \left(\alpha_{p q,\left\{n_{k}^{g}\right\}_{k=1}^{i}}-\gamma_{A}\right)} \\
& \times e^{j 2 \pi f_{\text {max }}^{B} t \cos \left(\phi_{p q,\left\{n_{k}^{g}\right\}_{k=1}^{i}}-\gamma_{B}\right)} \delta\left(\tau-\tau_{\left\{n_{k}^{g}\right\}_{k=1}^{i}}\right)(3
\end{aligned}
$$

where $N_{k}^{g}$ is the number of effective scatterers in the $k$ th local scattering area with respect to the $g$ th $i$-bounced component, $\left\{\chi_{p q, n_{k}^{g}}\right\}_{k=1}^{i}$ is the travel path of the $g$ th $i$-bounced waves through the $A_{p}-B_{q}$ link, $\left\{\tau_{n_{k}^{g}}\right\}_{k=1}^{i}$ denotes the time delay of the multipath components. The phases $\left\{\psi_{n_{k}}\right\}_{k=1}^{i}$ are independent and identically distributed (i.i.d.) random variables with uniform distributions over $[-\pi, \pi)$ and determined by scatterers $\left\{S_{n_{K}}\right\}_{k=1}^{i},\left\{X_{n_{k}}\right\}_{k=1}^{i}$ represents $X_{n_{1}}, X_{n_{2}}, X_{n_{3}}, \ldots, X_{n_{i}}$, and $\left\{\alpha_{p q, n_{k}^{g}}\right\}_{k=1}^{i}$ and $\left\{\phi_{p q, n_{k}^{g}}\right\}_{k=1}^{i}$ denote angles of arrival/departure of a $i$-bounced path with respect to Node $A$ and Node $B$, respectively. Here, $\eta_{p q}^{i g}$ is a energy-related parameter specifying how much the $g$ th $i$-bounced rays contribute to the total scattered power $\Omega_{p q} /\left(K_{p q}+1\right)$. Note that energy-related parameters satisfy $\sum_{i=1}^{I} \sum_{g=1}^{f_{I}(i)} \eta_{p q}^{i g}=1$.

It is clear that the proposed unified channel model framework in (1) can naturally include the impact of local scattering area on channel characteristics with the help of properly choosing $f_{I}(i) i$-bounced components. Note that parameter $f_{I}(i)$ is related to the number of related local scattering areas $I$, which is determined by physical environments, outdoor macro-cell, micro-cell, pico-cell, and indoor. This means by properly adjusting the parameter $I$, the proposed model 
framework is suitable for different basic cooperative environments. Furthermore, the proposed model framework can model multiple links with different degrees of link heterogeneity due to different application scenes (e.g., BS cooperation, MS cooperation, and relay cooperation) for a typical cooperative environment by simply adjusting the Ricean factor $K_{p q}$ and energy-related parameters $\eta_{p q}^{i g}$. How to properly set these key model parameters, i.e., $I, K_{p q}, \eta_{p q}^{i g}$, will be explained in the next section.

\section{A NEW MIMO GBSM FOR COOPERATIVE RELAY SySTEMS}

Without loss of generality, this section considers a wideband cooperative relay communication environment that includes three different links: BS-RS, RS-MS, and BS-MS, to implement the proposed cooperative MIMO channel model framework. Note that the designed cooperative MIMO GBSM can be easily extended to other cooperative MIMO scenarios with multiple relays. The RS can be another BS for BS cooperation or another MS for MS cooperation. In order to propose a generic cooperative MIMO GBSM that is suitable for the aforementioned 12 cooperative scenarios, we assume that the BS, RS, and MS are all surrounded by local scatterers. Fig. 2 shows the geometry of the proposed cooperative MIMO GBSM, combining the LoS components and scattered components. To keep the readability of Fig. 2, the LoS components are not shown. It is assumed that the BS, RS, and MS are all equipped with $A_{B}=A_{R}=A_{M}=2$ uniform linear antenna arrays. The local scattering environment is characterized by the effective scatterers located on circular rings. Suppose there are $N_{1}$ effective scatterers around the MS lying on a circular ring of radius $R_{1 n_{1}} \leq \xi_{n_{1}}^{M} \leq R_{1 n_{2}}$ and the $n_{1}$ th $\left(n_{1}=1, \ldots, N_{1}\right)$ effective scatterer is denoted by $S_{n_{1}}$. Similarly, assume there are $N_{2}$ effective scatterers around the RS lying on a circular ring of radius $R_{2 n_{1}} \leq \xi_{n_{2}}^{R} \leq R_{2 n_{2}}$ and the $n_{2}$ th $\left(n_{2}=1, \ldots, N_{2}\right)$ effective scatterer is denoted by $S_{n_{2}}$. For the local scattering area around BS, $N_{3}$ effective scatterers lie on a circular ring of radius $R_{3 n_{1}} \leq \xi_{n_{3}}^{B} \leq R_{3 n_{2}}$ and the $n_{3}$ th $\left(n_{3}=1, \ldots, N_{3}\right)$ effective scatterer is denoted by $S_{n_{3}}$. The parameters in Fig. 2 are defined in Table I.

As this paper only focuses on the investigation of multilink spatial correlations (not time or frequency correlations), we will neglect $t$ and $\tau$ in (1) for the proposed channel model framework to simplify notations. Due to the page limit, the spatial correlation properties between the BS-RS link and BSMS link are chosen as an example for detailed investigation. Therefore, in the following, we will show the channel gains of the BS-RS link and BS-MS link for the proposed cooperative MIMO GBSM.

\section{A. $B S-R S(M S) l i n k$}

The channel gain of the BS-RS(MS) link between antenna $p_{3}$ at the $\mathrm{BS}$ and antenna $p_{2}$ at the $\operatorname{RS}\left(p_{1}\right.$ at the $\left.\mathrm{MS}\right)$ can be expressed as

$$
h_{p_{3} p_{2}\left(p_{1}\right)}=h_{p_{3} p_{2}\left(p_{1}\right)}^{L o S}+\sum_{i=1}^{3} \sum_{g=1}^{f_{3}(i)} h_{p_{3} p_{2}\left(p_{1}\right)}^{i g}
$$

TABLE I

TABle I. Definition OF PARAmeters IN Fig. 2.

\begin{tabular}{|c|c|}
\hline$D_{1}, D_{2}, D_{3}$ & distances of BS-MS, RS-MS, and BS-RS, respectively \\
\hline $\begin{array}{l}R_{1 n_{1}}, R_{2 n_{1}} ; R_{1 n_{2}} \\
R_{2 n_{2}} ; R_{1 n_{3}}, R_{2 n_{3}}\end{array}$ & $\begin{array}{l}\min \text { and max radii of the circular rings around } \\
\text { the MS, RS and BS, respectively }\end{array}$ \\
\hline$\theta, \theta^{\prime}$ & $\begin{array}{l}\text { angles between the RS-MS link and BS-MS link, and } \\
\text { between the BS-RS link and BS-MS link, respectively }\end{array}$ \\
\hline$\delta_{1}, \delta_{2}, \delta_{3}$ & antenna element spacings of MS, RS and BS, respectively \\
\hline$\beta_{1}, \beta_{2}, \beta_{3}$ & $\begin{array}{l}\text { orientations of the MS, RS and RS antenna arrays in } \\
\text { the } x-y \text { plane (relative to the } x \text {-axis), respectively }\end{array}$ \\
\hline $\begin{array}{c}\alpha_{1 n_{i}}, \alpha_{2 n_{i}} \\
\quad \text { and } \alpha_{3 n_{i}}\end{array}$ & $\begin{array}{l}\text { azimuth angles of } S_{n_{i}} \text {-MS, } S_{n_{i}} \text {-RS, and } S_{n_{i}} \text {-BS links } \\
\text { in the x-y plane (relative to the x-axis), respectively }\end{array}$ \\
\hline$\xi_{n_{1}}^{B}, \xi_{n_{2}}^{B}, \xi_{n_{3}}^{B}$ & $\begin{array}{l}\text { distances } d\left(\mathrm{BS}, S_{n_{1}}\right), d\left(\mathrm{BS}, S_{n_{2}}\right), \text { and } \\
d\left(\mathrm{BS}, S_{n_{3}}\right), \text { respectively }\end{array}$ \\
\hline$\xi_{n_{1}}^{R}, \xi_{n_{2}}^{R}, \xi_{n_{3}}^{R}$ & $\begin{array}{l}\text { distances } d\left(\mathrm{RS}, S_{n_{1}}\right), d\left(\mathrm{RS}, S_{n_{2}}\right), \text { and } \\
d\left(\mathrm{RS}, S_{n_{3}}\right) \text {, respectively }\end{array}$ \\
\hline$\xi_{n_{1}}^{M}, \xi_{n_{2}}^{M}, \xi_{n_{3}}^{M}$ & $\begin{array}{l}\text { distances } d\left(\mathrm{MS}, S_{n_{1}}\right), d\left(\mathrm{MS}, S_{n_{2}}\right), \text { and } \\
d\left(\mathrm{MS}, S_{n_{3}}\right) \text {, respectively }\end{array}$ \\
\hline $\begin{array}{c}\varepsilon_{p_{i} n_{g}}\left(\varepsilon_{n g} p_{i}\right) \\
\varepsilon_{p_{i} p_{j}}, \text { and } \varepsilon_{n g} n_{k}\end{array}$ & $\begin{array}{l}\text { distances } d\left(p_{i}, S_{n_{g}}\right), d\left(p_{i}, p_{j}\right), \text { and } \\
d\left(S_{n_{g}}, S_{n_{k}}\right), \text { respectively }\end{array}$ \\
\hline
\end{tabular}

where $h_{p_{3} p_{2}}^{L o S}$ and $h_{p_{3} p_{1}}^{\operatorname{LoS}}$ denote the LoS component of the BSRS link and the BS-MS link, respectively, and $h_{p_{3} p_{2}}^{i g}$ and $h_{p_{3} p_{1}}^{i g}$ represent the $g$ th $i$-bounced component of the BS-RS link and the BS-MS link, respectively, with the following expressions

$$
\begin{aligned}
& h_{p_{3} p_{2}\left(p_{1}\right)}^{L o S}=\sqrt{\frac{K_{p_{3} p_{2}\left(p_{1}\right)} \Omega_{p_{3} p_{2}\left(p_{1}\right)}}{K_{p_{3} p_{2}\left(p_{1}\right)}+1}} e^{-j 2 \pi \lambda^{-1} \chi_{p_{3} p_{2}\left(p_{1}\right)}} \\
& h_{p_{3} p_{2}\left(p_{1}\right)}^{1 g}=\sqrt{\frac{\eta_{p_{3} p_{2}\left(p_{1}\right)}^{1 g} \Omega_{p_{3} p_{2}\left(p_{1}\right)}}{K_{p_{3} p_{2}\left(p_{1}\right)}+1}} \lim _{N_{g} \rightarrow \infty} \sum_{n_{g}=1}^{N_{g}} \frac{1}{\sqrt{N_{g}}} \\
& \times e^{j\left(\psi_{n_{g}}-2 \pi \lambda^{-1} \chi_{p_{3} p_{2}\left(p_{1}\right), n_{g}}\right)} \\
& h_{p_{3} p_{2}\left(p_{1}\right)}^{2 g}=\sqrt{\frac{\eta_{p_{3} p_{2}\left(p_{1}\right)}^{2 g} \Omega_{p_{3} p_{2}\left(p_{1}\right)}}{K_{p_{3} p_{2}\left(p_{1}\right)}+1}} \lim _{N_{g_{1}}, N_{g_{2}} \rightarrow \infty} \sum_{n_{g_{1}}, n_{g_{2}}=1}^{N_{g_{1}}, N_{g_{2}}} \\
& \times \frac{1}{\sqrt{N_{g_{1}} N_{g_{2}}}} e^{j\left(\psi_{n_{g_{1}}, n_{g_{2}}}-2 \pi \lambda^{-1} \chi_{\left.p_{3} p_{2}\left(p_{1}\right), n_{g_{1}, n_{g_{2}}}\right)}\right.} \\
& h_{p_{3} p_{2}\left(p_{1}\right)}^{31}=\sqrt{\frac{\eta_{p_{3} p_{2}\left(p_{1}\right)}^{31} \Omega_{p_{3} p_{2}\left(p_{1}\right)}}{K_{p_{3} p_{2}\left(p_{1}\right)}+1}} \lim _{N_{1}, N_{2}, N_{3} \rightarrow \infty} \sum_{n_{1}, n_{2}, n_{3}=1}^{N_{1}, N_{2}, N_{3}} \\
& \times \frac{1}{\sqrt{N_{1} N_{2} N_{3}}} e^{j\left(\psi_{n_{1}, n_{2}, n_{3}}-2 \pi \lambda^{-1} \chi_{p_{3} p_{2}\left(p_{1}\right), n_{1}, n_{2}, n_{3}}\right)}
\end{aligned}
$$

where $g=1,2,3,\left\{g_{1}, g_{2}\right\}=\{3,2\}$ for $g=1,\left\{g_{1}, g_{2}\right\}=\{3,1\}$ for $g=2$, and $\left\{g_{1}, g_{2}\right\}=\{1,2\}$ for $g=3$. In (5)-(8), $\chi_{p_{3} p_{2}\left(p_{1}\right)=}$ $\varepsilon_{p_{3} p_{2}\left(p_{1}\right)}, \chi_{p_{3} p_{2}\left(p_{1}\right), n_{g}}=\varepsilon_{p_{3} n_{g}}+\varepsilon_{n_{g} p_{2}\left(p_{1}\right)}, \chi_{p_{3} p_{2}\left(p_{1}\right), n_{g_{1}, n_{g_{2}}}}=$ $\varepsilon_{p_{3} n_{g_{1}}}+\varepsilon_{n_{g_{1}} n_{g_{2}}}+\varepsilon_{n_{g_{2}} p_{2}\left(p_{1}\right)}$, and $\chi_{p_{3} p_{2}\left(p_{1}\right), n_{1}, n_{2}, n_{3}}=\varepsilon_{p_{3} n_{3}}+$ $\varepsilon_{n_{3} n_{1}\left(n_{2}\right)}+\varepsilon_{n_{1} n_{2}}+\varepsilon_{n_{2}\left(n_{1}\right) p_{2}\left(p_{1}\right)}$ are the travel times of the waves through the link $B_{p_{3}}-R_{p_{2}}\left(M_{p_{1}}\right), B_{p_{3}}-S_{n_{g}}-R_{p_{2}}\left(M_{p_{1}}\right)$, $B_{p_{3}}-S_{n_{g_{1}}}-S_{n_{g_{2}}}-R_{p_{2}}\left(M_{p_{1}}\right)$, and $B_{p_{3}}-S_{n_{3}}-S_{n_{1}}\left(S_{n_{2}}\right)-$ $S_{n_{2}}\left(S_{n_{1}}\right)-R_{p_{2}}\left(M_{p_{1}}\right)$, respectively. The symbols $K_{p_{3} p_{2}\left(p_{1}\right)}$ and $\Omega_{p_{3} p_{2}\left(p_{1}\right)}$ designate the Ricean factor and the total power of the BS-RS(MS) link, respectively. Parameters $\eta_{p_{3} p_{2}\left(p_{1}\right)}^{1 g}$, $\eta_{p_{3} p_{2}\left(p_{1}\right)}^{2 g}$, and $\eta_{p_{3} p_{2}\left(p_{1}\right)}^{31}$ specify how much the single-, double-, and triple-bounced rays contribute to the total scattered power $\Omega_{p_{3} p_{2}\left(p_{1}\right)} /\left(K_{p_{3} p_{2}\left(p_{1}\right)}+1\right)$ with $\sum_{g=1}^{3}\left(\eta_{p_{3} p_{2}\left(p_{1}\right)}^{1 g}+\eta_{p_{3} p_{2}\left(p_{1}\right)}^{2 g}\right)+$ $\eta_{p_{3} p_{2}\left(p_{1}\right)}^{31}=1$. The phases $\psi_{n_{g}}, \psi_{n_{g_{1}}, n_{g_{2}}}$, and $\psi_{n_{1}, n_{2}, n_{3}}$ are i.i.d. random variables with uniform distributions over $[-\pi, \pi)$.

From Fig. 2 and based on the normally used assumption $\min \left\{D_{1}, D_{2}, D_{3}\right\} \gg \max \left\{\delta_{1}, \delta_{2}, \delta_{3}\right\}$ [22] and the application 


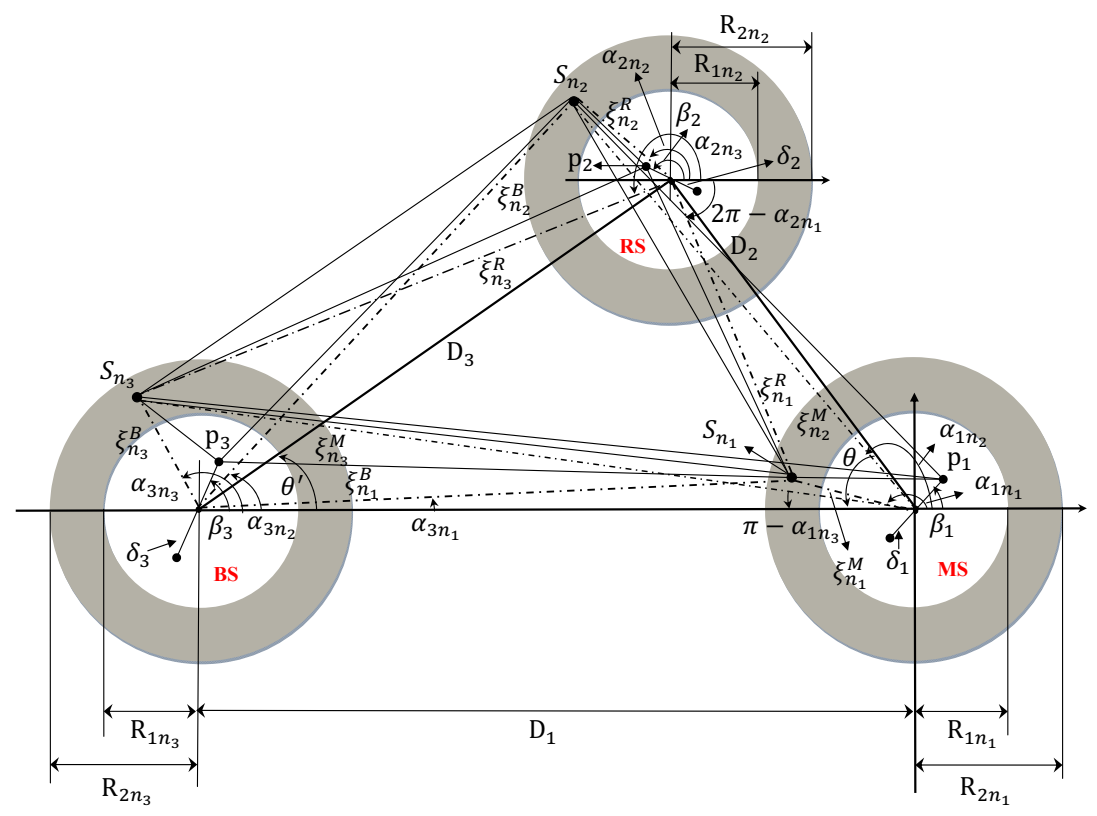

Fig. 2. The proposed cooperative MIMO GBSM.

of the law of cosines in appropriate triangles, the distances in (5)-(8) can be expressed as $\varepsilon_{p_{3} p_{2}} \approx D_{3}-\frac{\delta_{3}}{2} \cos \left(\beta_{3}-\theta^{\prime}\right)+$ $\frac{\delta_{2}}{2} \cos \left(\beta_{2}-\theta^{\prime}\right), \varepsilon_{p_{3} p_{1}} \approx D_{1}-\frac{\delta_{3}}{2} \cos \beta_{3}+\frac{\delta_{1}}{2} \cos \left(\beta_{1}\right), \varepsilon_{p_{3} n_{g}} \approx$ $\xi_{n_{g}}^{B}-\frac{\delta_{3}}{2} \cos \left(\beta_{3}-\alpha_{1 n_{g}}\right), \varepsilon_{n_{g} p_{2}} \approx \xi_{n_{g}}^{R}-\frac{\delta_{2}}{2} \cos \left(\beta_{2}-\alpha_{2 n_{g}}\right)$, $\varepsilon_{n_{g} p_{1}} \approx \xi_{n_{g}}^{M}-\frac{\delta_{1}}{2} \cos \left(\beta_{1}-\phi_{n_{g}}\right), \varepsilon_{n_{1} n_{2}}=\left[\left(\xi_{n_{1}}^{R}\right)^{2}+\left(\xi_{n_{2}}^{R}\right)^{2}-\right.$ $\left.2 \xi_{n_{1}}^{R} \xi_{n_{2}}^{R} \cos \left(\alpha_{2 n_{1}}-\alpha_{2 n_{2}}\right)\right]^{1 / 2}$, and $\varepsilon_{n_{3} n_{o}}=\left[\left(\xi_{n_{3}}^{B}\right)^{2}+\left(\xi_{n_{o}}^{B}\right)^{2}-\right.$ $\left.2 \xi_{n_{3}}^{B} \xi_{n_{o}}^{B} \cos \left(\alpha_{3 n_{3}}-\alpha_{1 n_{e}}\right)\right]^{1 / 2}$, where $\xi_{n_{1}}^{B}=\left[D_{1}^{2}+\left(\xi_{n_{1}}^{M}\right)^{2}+\right.$ $\left.2 D_{1} \xi_{n_{1}}^{M} \cos \alpha_{1 n_{1}}\right]^{1 / 2}, \xi_{n_{1}}^{R}=\left[D_{2}^{2}+\left(\xi_{n_{1}}^{M}\right)^{2}+2 D_{2} \xi_{n_{1}}^{M} \cos \left(\alpha_{1 n_{1}}+\right.\right.$ $\theta)]^{1 / 2}, \xi_{n_{2}}^{B}=\left[D_{3}^{2}+\left(\xi_{n_{2}}^{R}\right)^{2}+2 D_{3} \xi_{n_{2}}^{R} \cos \left(\alpha_{2 n_{2}}-\theta^{\prime}\right)\right]^{1 / 2}, \xi_{n_{3}}^{R}=$ $\left[D_{3}^{2}+\left(\xi_{n_{3}}^{B}\right)^{2}-2 D_{3} \xi_{n_{3}}^{B} \cos \left(\alpha_{3 n_{3}}-\theta^{\prime}\right)\right]^{1 / 2}, \xi_{n_{2}}^{R} \in\left[R_{1 n_{2}}, R_{2 n_{2}}\right]$, $\xi_{n_{3}}^{B} \in\left[R_{1 n_{3}}, R_{2 n_{3}}\right], \varrho=1,2$, and $g=1,2,3$. Note that the AoD $\alpha_{3 n_{1}}, \alpha_{3 n_{2}}, \alpha_{3 n_{3}}$ and AoA $\alpha_{2 n_{1}}, \alpha_{2 n_{2}}, \alpha_{2 n_{3}}$ are independent for double- and triple-bounced rays, while they are interdependent for single-bounced rays. It is worth highlighting that scatterers $S_{n_{g}}$ around MS, RS, and BS are relevant to the angles $\alpha_{1 n_{1}}, \alpha_{2 n_{2}}$, and $\alpha_{3 n_{3}}$, respectively. Therefore, all other AoDs and AoAs have to be related to the aforementioned three key angles. By following the general method given in [22], the relationship of the key angles with other AoAs and AoDs of BS-RS link can be obtained as: $\sin \alpha_{3 n_{1}}=\frac{\xi_{n_{1}}^{M}}{\xi_{n_{1}}^{B}} \sin \alpha_{1 n_{1}}, \sin \left(\alpha_{2 n_{1}}+\theta\right)=\frac{\xi_{n_{1}}^{M}}{\xi_{n_{1}}^{R}} \sin \left(\theta+\alpha_{1 n_{1}}\right)$, $\sin \left(\alpha_{3 n_{2}}-\theta^{\prime}\right)=\frac{\xi_{n_{2}}^{R}}{\xi_{n_{2}}^{B}} \sin \left(\alpha_{2 n_{2}}-\theta^{\prime}\right)$, and $\sin \left(\alpha_{2 n_{3}}-\theta^{\prime}\right)=$ $\frac{\xi_{n_{3}}^{B}}{\xi_{n_{3}}^{R}} \sin \left(\alpha_{3 n_{3}}-\theta^{\prime}\right)$. The channel gain of the RS-MS link can be similarly obtained and omitted here for brevity.

In the literature, different scatterer distributions have been proposed to characterize the key angles $\alpha_{1 n_{1}}, \alpha_{2 n_{2}}$, and $\alpha_{3 n_{3}}$, such as the uniform and Gaussian PDFs [23]. In this paper, the von Mises PDF [24] is used, which is more generic and can approximate all the aforementioned PDFs [22]. The von Mises PDF is defined as $f(\phi) \triangleq \exp [k \cos (\phi-\mu)] /\left[2 \pi I_{0}(k)\right]$, where $\phi \in[-\pi, \pi), I_{0}(\cdot)$ is the zeroth-order modified Bessel function of the first kind, $\mu \in[-\pi, \pi)$ accounts for the mean value of the angle $\phi$, and $k(k \geq 0)$ is a real-valued parameter that controls the angle spread of the angle $\phi$. In this paper, for the key angles, i.e., the $\alpha_{1 n_{1}}, \alpha_{2 n_{2}}$, and $\alpha_{3 n_{3}}$, we use appropriate parameters ( $\mu$ and $k$ ) of the von Mises PDF as $\mu_{1}$ and $k_{1}, \mu_{2}$ and $k_{2}$, and $\mu_{3}$ and $k_{3}$, respectively.

\section{B. Adjustment of Key Model Parameters}

The proposed cooperative MIMO GBSM is adaptable to the above mentioned 12 cooperative scenarios for this interested typical cooperative MIMO environment by adjusting key model parameters. From previous section, we know that these important model parameters are the number of local scattering environment $I$, Ricean factors $K_{p_{3} p_{2}}, K_{p_{3} p_{1}}, K_{p_{2} p_{1}}$ that denotes the Ricean factor of the RS-MS link, and energyrelated parameters $\eta_{p_{3} p_{2}}^{i g}, \eta_{p_{3} p_{1}}^{i g}$, and $\eta_{p_{2} p_{1}}^{i g}$ that is the energyrelated parameter of the RS-MS link. The parameter setting of $I$ is basically based on basic scenario. For outdoor micro-cell, pico-cell, and indoor scenarios, we assume that the BS, RS, and MS are all surrounded by local scattering area as shown in Fig. 2 and thus $I=3$ in this case. For outdoor Marcocell scenario, the BS is free of scatterers and thus $I=2$. In this case, the channel model can also be obtained from the proposed model by setting the energy-related parameters related to the local scatterers around BS equal to zero, e.g., for BS-RS link, the channel model can be obtained from (4) by setting $\eta_{p_{3} p_{2}}^{13}=\eta_{p_{3} p_{2}}^{21}=\eta_{p_{3} p_{2}}^{22}=\eta_{p_{3} p_{2}}^{31}=0$. For outdoor macro-cell BS cooperation scenario, RS actually represents the other BS, symbolled as BS2, and thus is free of scatterers as well. In this case, we have the currently most mature cooperative MIMO scheme: CoMP and the number of local scattering area $I=1$. Similarly, the channel model with $I=1$ can also be obtained from the proposed model by setting the energy-related parameters related to the local scatterers around $\mathrm{BS}$ and $\mathrm{RS}(\mathrm{BS} 2)$ equal to zero. It is clear that the 
proposed GBSM can be adaptable to different basic scenarios by setting relevant energy-related parameters equal to zero. Therefore, the key model parameters of the proposed GBSM actually are reduced as the Ricean factors and energy-related parameters. The basic criterion of setting these key model parameters is summarized as following: the longer distance of the link and/or the higher the LSD, the smaller the Ricean factors and the larger the energy-related parameters of multibounced components, i.e., the multi-bounced components bear more energy than single-bounced components. Since the local scattering area is highly related to the degree of link heterogeneity in cooperative MIMO systems as presented in [18], [21], the LSD significantly affects the channel characteristics and should be investigated. In general, the higher the LSD, the lower the possibility that the devices (BS/MS/RS) share the same scatterers. In this case, the cooperative MIMO environments present lower environment similarity. Therefore, the higher the LSD, the lower the environment similarity.

For macro-cell scenarios, the Ricean factor $K_{p_{3} p_{1}}$ is very small or even close to zero due to the large distance $D_{1}$. Under the condition of BS cooperation scenes, Ricean factor $K_{p_{2} p_{1}}$ is similar to $K_{p_{3} p_{1}}$ due to the similar distances of $D_{1}$ and $D_{2}$. While the BS-RS link is disappeared and replaced by wired link. In this case, we only have single bounced components, i.e., $\eta_{p_{3} p_{1}}^{11}$ and $\eta_{p_{2} p_{1}}^{11}$. For $\mathrm{MS} / \mathrm{RS}$ cooperation scenes, RS/MS actually represents the other MS/RS and is symbolled as MS2/RS2. In this case, Ricean factor $K_{p_{3} p_{2}}$ is similar to $K_{p_{3} p_{1}}$ due to the similar distances of $D_{1}$ and $D_{3}$. Since the large value of distances $D_{1}$ and $D_{3}$, for the BS-MS/BR2 link and BS-MS2/RS link, the impact of LSD on channel characteristics is small and in general the doublebounced rays bear more energy than single-bounced rays, i.e., $\left\{\eta_{p_{3} p_{1}}^{21}, \eta_{p_{3} p_{2}}^{21}\right\}>\left\{\eta_{p_{3} p_{1}}^{11}, \eta_{p_{3} p_{1}}^{12}, \eta_{p_{3} p_{2}}^{11}, \eta_{p_{3} p_{2}}^{12}\right\}$. While for the MS/RS2-MS2/RS link, due to the small distance of $D_{2}$ the impact of LSD is significant. For a low LSD, the scatterers are sparse and thus more likely single-bounced rays rather than double-bounced rays, i.e., $\left\{\eta_{p_{2} p_{1}}^{11}, \eta_{p_{2} p_{1}}^{12}\right\}>\eta_{p_{2} p_{1}}^{21}$, and Ricean factor $K_{p_{2} p_{1}}$ is large. For a high LSD, the doublebounced components bear more energy than single-bounced components, i.e., $\eta_{p_{2} p_{1}}^{21}>\left\{\eta_{p_{2} p_{1}}^{11}, \eta_{p_{2} p_{1}}^{12}\right\}$ and Ricean factor $K_{p_{2} p_{1}}$ is smaller than that in the low LSD. Similar to macrocell scenarios, the key model parameters setting for other 9 cooperative scenarios with the consideration of different LSDs can be easily obtained by following the aforementioned basic criterion and thus omits here for brevity.

\section{Multi-link Spatial Correlation Functions}

In this section, based on the proposed cooperative MIMO GBSM and considering the spatial correlation between the BS-RS link and the BS-MS link as an example, we will derive the multi-link spatial correlation functions for nonisotropic scattering cooperative MIMO environments. The normalized spatial correlation function between any two links characterized by channel gains $h_{p q}$ and $h_{p^{\prime} q^{\prime}}$, respectively, is defined as [25], [26]

$$
\rho_{p q, p^{\prime} q^{\prime}}=\frac{\mathbf{E}\left[h_{p q} h_{p^{\prime} q^{\prime}}^{*}\right]}{\sqrt{\Omega_{p q} \Omega_{p^{\prime} q^{\prime}}}}
$$

where $(\cdot)^{*}$ denotes the complex conjugate operation, $\mathbf{E}[\cdot]$ is the statistical expectation operator, $p, p^{\prime} \in\left\{1,2, \ldots, M_{T}\right\}$, and $q, q^{\prime} \in\left\{1,2, \ldots, M_{R}\right\}$. Substituting (4) into (9), we have the correlation function between BS-RS link and BS-MS link as $\rho_{p_{3} p_{2}, p_{3}^{\prime} p_{1}}=\rho_{p_{3} p_{2}, p_{3}^{\prime} p_{1}}^{L o S}+\sum_{g=1}^{3}\left(\rho_{p_{3} p_{2}, p_{3}^{\prime} p_{1}}^{1 g}+\rho_{p_{3} p_{2}, p_{3}^{\prime} p_{1}}^{2 g}\right)+\rho_{p_{3} p_{2}, p_{3}^{\prime} p_{1}}^{31}$

with

$$
\rho_{p_{3} p_{2}, p_{3}^{\prime} p_{1}}^{L o S}=\sqrt{\frac{K_{p_{3} p_{2}} K_{p_{3}^{\prime} p_{1}}}{\left(K_{p_{3} p_{2}}+1\right)\left(K_{p_{3}^{\prime} p_{1}}+1\right)}} e^{j 2 \pi \lambda^{-1}\left(\chi_{p_{3}^{\prime} p_{1}}-\chi_{p_{3} p_{2}}\right)}
$$

$$
\begin{aligned}
\rho_{p_{3} p_{2}, p_{3}^{\prime} p_{1}}^{1 g} & =\sqrt{\frac{\eta_{p_{3} p_{2}}^{1 g} \eta_{p_{3}^{\prime} p_{1}}^{1 g}}{\left(K_{p_{3} p_{2}}+1\right)\left(K_{p_{3}^{\prime} p_{1}}+1\right)}} \int_{-\pi}^{\pi} \int_{R_{1 n_{g}}}^{R_{2 n_{g}}} \\
& \times e^{j 2 \pi \lambda^{-1}\left(\chi_{p_{3}^{\prime} p_{1}, g}-\chi_{p_{3} p_{2}, g}\right)} Q_{g} f\left(\emptyset_{g}\right) d \emptyset_{g} d \Im_{g}
\end{aligned}
$$

$$
\begin{aligned}
\rho_{p_{3} p_{2}, p_{3}^{\prime} p_{1}}^{2 g} & =\sqrt{\frac{\eta_{p_{3} p_{2}}^{2 g} \eta_{p_{3}^{\prime} p_{1}}^{2 g}}{\left(K_{p_{3} p_{2}}+1\right)\left(K_{p_{3}^{\prime} p_{1}}+1\right)}} \int_{-\pi}^{\pi} \int_{-\pi}^{\pi} \int_{R_{1 n_{g_{1}}}}^{R_{2 n_{g_{1}}}} \\
& \int_{R_{1 n_{g_{2}}}{ }_{2 n_{g_{2}}}^{Q_{n_{1} g_{2}}} f\left(\emptyset_{g_{1}}\right) f\left(\emptyset_{g_{2}}\right) e^{j 2 \pi \lambda^{-1} \chi_{p_{3}^{\prime} p_{1}, g_{1}, g_{2}}}} \\
& \times e^{-j 2 \pi \lambda^{-1} \chi_{p_{3} p_{2}, g_{1}, g_{2}} d \emptyset_{g_{1}} d \emptyset_{g_{2}} d \Im_{g_{1}} d \Im_{g_{2}}}
\end{aligned}
$$

$$
\begin{aligned}
\rho_{p_{3} p_{2}, p_{3}^{\prime} p_{1}}^{31} & =\sqrt{\frac{\eta_{p_{3} p_{2}}^{31} \eta_{p_{3}^{\prime} p_{1}}^{31}}{\left(K_{p_{3} p_{2}}+1\right)\left(K_{p_{3}^{\prime} p_{1}}+1\right)}} \int_{-\pi}^{\pi} \int_{-\pi}^{\pi} \int_{-\pi}^{\pi} \\
& \int_{R_{1 n_{1}}}^{R_{2 n_{1}}} \int_{R_{1 n_{2}}}^{R_{2 n_{2}}} \int_{R_{1 n_{3}}}^{R_{2 n_{3}}} Q_{123} e^{j 2 \pi \lambda^{-1}}\left(\chi_{p_{3}^{\prime} p_{1}, 1,2,3}-\chi_{p_{3} p_{2}, 1,2,3}\right) \\
& \times f\left(\emptyset_{1}\right) f\left(\emptyset_{2}\right) f\left(\emptyset_{3}\right) d \emptyset_{1} d \emptyset_{2} d \emptyset_{3} d \Im_{1} d \Im_{2} d \Im_{3}
\end{aligned}
$$

where $\left\{\emptyset_{g}\right\}_{g=1}^{3}=\left\{\phi_{1}, \alpha_{2,2}, \alpha_{1,3}\right\}$ are the continuous expressions of the discrete expressions of angles $\alpha_{1 n_{1}}, \alpha_{2 n_{2}}, \alpha_{3 n_{3}}$, respectively, and $\varepsilon_{p_{3}^{\prime} n_{g}} \approx \xi_{n_{g}}^{B}+$ $\frac{\delta_{3}}{2} \cos \left(\beta_{3}-\alpha_{1 n_{g}}\right), \quad \chi_{p_{3}^{\prime} p_{1}, g}=\chi_{p_{3}^{\prime} p_{1}, n_{g}}, \quad \chi_{p_{3} p_{2}, g}=$ $\chi_{p_{3} p_{2}, n_{g}}, \quad \chi_{p_{3}^{\prime} p_{1}, g_{1}, g_{2}}=\chi_{p_{3}^{\prime} p_{1}, n_{g_{1}}, n_{g_{2}}}, \quad \chi_{p_{3} p_{2}, g_{1}, g_{2}}=$ $\chi_{p_{3} p_{2}, n_{g_{1}}, n_{g_{2}}}, \chi_{p_{3}^{\prime} p_{1}, 1,2,3}=\chi_{p_{3}^{\prime} p_{1}, n_{1}, n_{2}, n_{3}}$, and $\chi_{p_{3} p_{2}, 1,2,3}=$ $\chi_{p_{3} p_{2}, n_{1}, n_{2}, n_{3}}$ with $\alpha_{1 n_{1}}, \alpha_{2 n_{2}}$, and $\alpha_{3 n_{3}}$ being replaced by $\phi_{1}, \alpha_{2,2}$, and $\alpha_{1,3}$, respectively. Parameters $f\left(\emptyset_{g}\right)=$ $\exp \left[k_{g} \cos \left(\emptyset_{g}-\mu_{g}\right)\right] /\left[2 \pi I_{0}\left(k_{g}\right)\right],\left\{\Im_{g}\right\}_{g=1}^{3}=\left\{\xi_{n_{1}}^{M}, \xi_{2 n_{2}}^{R}, \xi_{1 n_{3}}^{B}\right\}$, $Q_{g}=\frac{2 \Im_{n_{g}}}{R_{2 n_{g}}^{2}-R_{1 n_{g}}^{2}}, Q_{g_{1} g_{2}}=\frac{4 \Im_{n_{g_{1}}} \Im_{n_{g_{2}}}}{\left(R_{2 n_{g_{1}}}^{2}-R_{1 n_{g_{1}}}^{2}\right)\left(R_{2 n_{g_{2}}}^{2}-R_{1 n_{g_{2}}}^{2}\right)}$, $Q_{123}=\frac{8 \Im_{n_{1}} \Im_{n_{2}} \Im_{n_{3}}}{\left(R_{2 n_{1}}^{2}-R_{1 n_{1}}^{2}\right)\left(R_{2 n_{2}}^{2}-R_{1 n_{2}}^{2}\right)\left(R_{2 n_{3}}^{2}-R_{1 n_{3}}^{2}\right)}$, and parameters $g, g_{1}$, and $g_{2}$ are the same as the ones in (5)-(8). Note that in (27) other correlation terms are equal to zero and thus omitted. These omitted correlation terms contain the integral of random phases $\psi_{n_{g}}, \psi_{n_{g_{1}}, n_{g_{2}}}$, or $\psi_{n_{1}, n_{2}, n_{3}}$. Since the random phases fulfill the uniform distribution over the range of $[\pi,-\pi)$, the integral of the random phases in the range of $[\pi,-\pi)$ is equal to zero. Therefore, other correlation terms with the value of zero are omitted in (27). Other two multi-link correlation functions can be similarly derived and thus omitted here for brevity. 


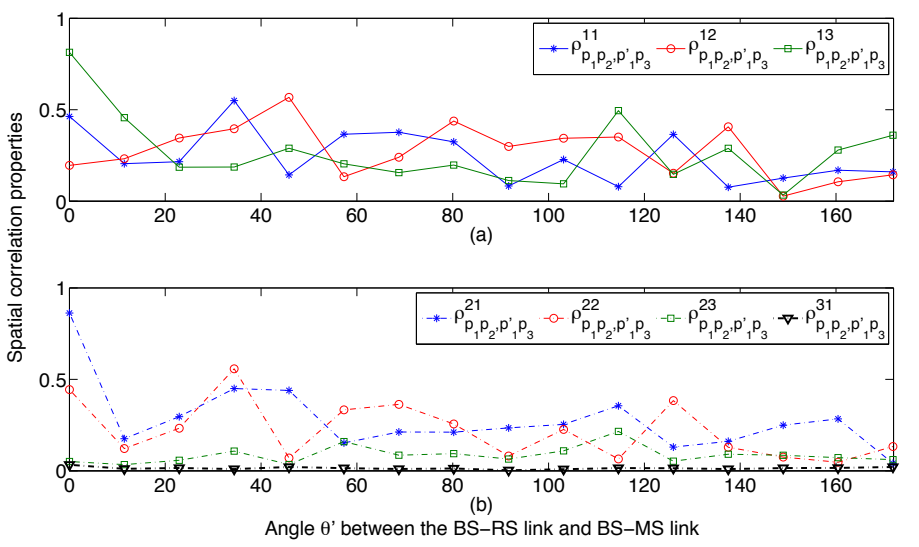

Fig. 3. Absolute values of spatial correlation functions between the BSRS link and BS-MS link for (a) the single-bounced components and (b) the double- and triple-bounce components.

\section{NumERiCAL RESUlts AND ANALYSiS}

In this section, the derived spatial correlation properties between the BS-RS link and BS-MS link will be numerically analyzed in detail. The parameters for the following numerical results are listed here or specified otherwise: $f=2.4 \mathrm{GHz}$, $D_{1}=D_{3}=100 \mathrm{~m}, R_{1 n_{1}}=R_{1 n_{2}}=R_{1 n_{3}}=5 \mathrm{~m}$, $R_{2 n_{1}}=R_{2 n_{2}}=R_{2 n_{3}}=50 \mathrm{~m}, \delta_{3}=\delta_{2}=\delta_{1}=0, \beta_{3}=30^{\circ}$, $\beta_{2}=\beta_{1}=60^{\circ}, K_{p_{3} p_{2}}=K_{p_{3}^{\prime} p_{1}}=0, k_{1}=k_{2}=k_{3}=10$, $\mu_{1}=120^{\circ}, \mu_{2}=300^{\circ}$, and $\mu_{3}=60^{\circ}$.

In Fig. 3, we present the spatial correlation properties of all scattered components in (10) with parameters $\delta_{3}=\delta_{2}=$ $\delta_{1}=3 \lambda$ and $k_{1}=k_{2}=k_{3}=3$. Fig. 3 clearly depicts that the spatial correlation properties vary significantly for different scattered components. More importantly, we notice that the scattered component that includes more bounced rays expresses lower spatial correlation properties. This is because with more bounced rays, the component is related to more local scattering areas and thus easier experiences higher degree of link heterogeneity, resulting in lower link similarity for this component.

To validate the utility of the proposed cooperative MIMO channel model, Fig. 4 investigates the spatial correlation properties of the proposed cooperative MIMO GBSM in (10). Without loss of any generality, the outdoor macro-cell MS cooperation scenario is chosen for further investigation. As shown in Fig. 4, three different LSD conditions are considered with parameters $\delta_{3}=\delta_{2}=\delta_{1}=3 \lambda$, i.e., high LSD, low LSD, and mixed LSD.

For outdoor macro-cell MS cooperation scenario, the BS is free of scatterers and the RS actually represents the other MS, symbolled as MS2. Therefore, we have the energy-related parameters related to the local scatterers around BS to be equal to zero, i.e., $\eta_{p_{3} p_{2}}^{13}=\eta_{p_{3}^{\prime} p_{1}}^{13}=\eta_{p_{3} p_{2}}^{21}=\eta_{p_{3}^{\prime} p_{1}}^{21}=\eta_{p_{3} p_{2}}^{22}=$ $\eta_{p_{3}^{\prime} p_{1}}^{22}=\eta_{p_{3} p_{2}}^{31}=\eta_{p_{3}^{\prime} p_{1}}^{31}=0$, and assume $D_{1}=D_{3}=1500 \mathrm{~m}$ and $K_{p_{3} p_{2}}=K_{p_{3}^{\prime} p_{1}}=0$ due to the large distance among BS, MS, and MS2. Considering the basic criterion of setting key model parameters expressed in Section III, we choose the other energy-related parameters as: $\eta_{p_{3} p_{2}}^{11}=\eta_{p_{3}^{\prime} p_{1}}^{11}=\eta_{p_{3} p_{2}}^{12}=$ $\eta_{p_{3}^{\prime} p_{1}}^{12}=0.05$ and $\eta_{p_{3} p_{2}}^{23}=\eta_{p_{3}^{\prime} p_{1}}^{23}=0.9$ for high LSD, and

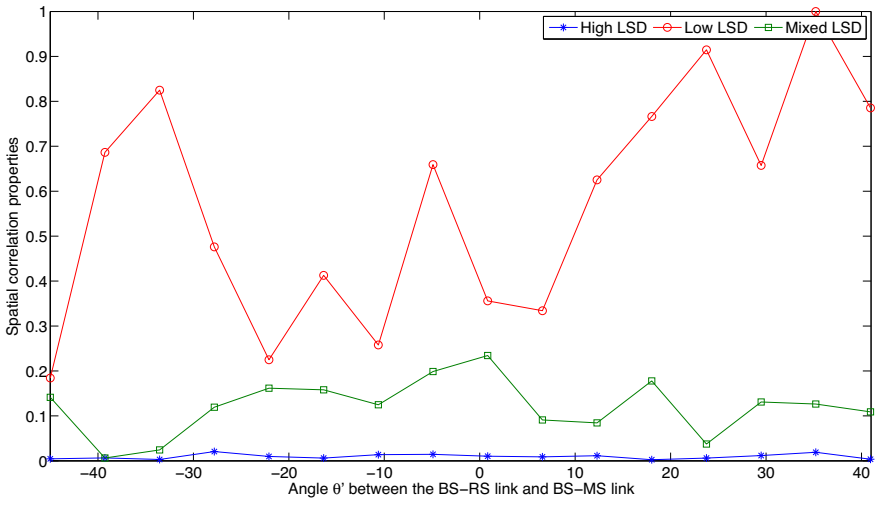

Fig. 4. Absolute values of spatial correlation functions between the BS-RS link and BS-MS link for the outdoor macro-cell MS cooperation scenario with different LSDs.

$\eta_{p_{3} p_{2}}^{11}=\eta_{p_{3}^{\prime} p_{1}}^{11}=\eta_{p_{3} p_{2}}^{12}=\eta_{p_{3}^{\prime} p_{1}}^{12}=0.2$ and $\eta_{p_{3} p_{2}}^{23}=\eta_{p_{3}^{\prime} p_{1}}^{23}=$ 0.6 for low LSD. While for mixed LSD case, we consider the scenario that the local scattering area around MS presents low LSD and the one around MS2 shows high LSD, and thus assume the energy-related parameters as: $\eta_{p_{3}^{\prime} p_{1}}^{11}=\eta_{p_{3}^{\prime} p_{1}}^{12}=$ $0.2, \eta_{p_{3}^{\prime} p_{1}}^{23}=0.6, \eta_{p_{3} p_{2}}^{11}=\eta_{p_{3} p_{2}}^{12}=0.1$, and $\eta_{p_{3} p_{2}}^{23}=0.8$. In general, the higher the LSD, the more distributed and larger size the local scattering area, and thereby the smaller the value of $k_{g}$ and the larger the value of $R_{2 n_{g}}-R_{1 n_{g}}(g=1,2,3)$. Therefore, we have the following environment parameters as: $k_{1}=k_{2}=1, R_{1 n_{1}}=R_{1 n_{2}}=5 \mathrm{~m}$, and $R_{2 n_{1}}=R_{2 n_{2}}=200 \mathrm{~m}$ for high LSD; $k_{1}=k_{2}=10, R_{1 n_{1}}=R_{1 n_{2}}=5 \mathrm{~m}$, and $R_{2 n_{1}}=R_{2 n_{2}}=20 \mathrm{~m}$ for low LSD; and $k_{1}=10, k_{2}=2$, $\mu_{1}=60^{\circ}, \mu_{2}=120^{\circ}, R_{1 n_{1}}=R_{1 n_{2}}=5 \mathrm{~m}, R_{2 n_{1}}=20 \mathrm{~m}$, and $R_{2 n_{2}}=100 \mathrm{~m}$ for mixed LSD.

Fig. 4 clearly shows that the LSD significantly affects the spatial correlation properties. It is observed that the higher the LSD, the lower the spatial correlation properties. This is because that with a higher LSD, the local scattering area is more distributed and presents larger size, resulting in the received power comes from many different directions. More importantly, from the observation in Fig. 3 and based on the constraints of the energy-related parameters for cooperative scenarios with different LSDs, we know that with a higher LSD, the multi-bounced components bear more energy than single-bounced components and thus the corresponding cooperative environment has a higher possibility to reveal a high degree of link heterogeneity, i.e., a low degree of environment similarity. Therefore, the above conclusion based on Fig. 4 is consistent with our intuition that a low degree of environment similarity results in low multi-link spatial correlations.

\section{CONCLUSIONS}

This paper has proposed a novel unified cooperative MIMO channel model framework, from which a novel GBSM has been further derived. The proposed multiple-ring GBSM is sufficiently generic and adaptable to a wide variety of cooperative MIMO propagation scenarios. More importantly, the proposed GBSM is the first model that is capable of investigating the impact of LSD on channel statistics. From the proposed GBSM, the multi-link spatial correlations have been derived and numerically evaluated. Numerical results have 
shown that the LSD has great impact on multi-link spatial correlation properties. It has also been demonstrated that a high multi-link spatial correlation may exist if the underlying propagation environments have low LSDs.

\section{REFERENCES}

[1] K. Fazel and S. Kaiser, Multi-Carrier and Spread Spectrum Systems: From OFDM and MC-CDMA to LTE and WiMAX, 2nd ed. John Wiley \& Sons, 2008.

[2] K. Yang, S. Ou, H. Chen, and J. He. "A multihop peer communication protocol with fairness guarantee for IEEE 802.16 based vehicular networks," IEEE Trans. Veh. Techno., vol. 56, no. 6, pp. 3358-3370, Nov. 2007.

[3] K. Yang, S. Ou, K. Guild, and H.-H. Chen. "Convergence of Ethernet PON and IEEE 802.16 broadband access networks and its QoS-aware dynamic bandwidth allocation scheme," IEEE J. Sel. Areas in Commun., vol. 27, no. 2, pp. 101-116, Feb. 2009.

[4] G. J. Foschini and M. J. Gans, "On limits of wireless communications in a fading environment when using multiple antennas," Wireless Personal Commun., vol. 6, no. 2, pp. 311-335, 1998.

[5] C.-X. Wang, X. Hong, H. Wu, and W. Xu, "Spatial temporal correlation properties of the $3 \mathrm{GPP}$ spatial channel model and the Kronecker MIMO channel model," EURASIP J. Wireless Commun. and Networking, vol. 2007, Article ID 39871, 9 pages, 2007. doi:10.1155/2007/39871.

[6] M. Mahinthan, X. Shen, and K. Naik, "Cooperative fair scheduling for the downlink of CDMA cellular networks," IEEE Trans. Veh. Techno., vol. 56, no. 4, pp. 1749-1760, Jul. 2007.

[7] V. Mahinthan, J. W. Mark, and X. Shen, "Performance analysis and power allocation for M-QAM cooperative diversity systems," IEEE Trans. Wireless Commun., vol. 9, no. 3, pp. 1237-1247, Mar. 2010.

[8] Z. Han, X. Zhang, and H. V. Poor, "High performance cooperative transmission protocols based on multiuser detection and network coding," IEEE Trans. Wireless Commun., vol. 8, no. 5, pp. 41-45, May 2009.

[9] C.-X. Wang, X. Cheng, and D. I. Laurenson, "Vehicle-to-vehicle channel modeling and measurements: recent advances and future challenges", IEEE Commun. Mag., vol. 47, no. 11, pp. 96-103, Nov. 2009.

[10] J. Karedal, A. Johansson, F. Tufvesson, and A. Molisch, "A measurement-based fading model for wireless personal area networks," IEEE Trans. Wireless Commun., vol. 7, no. 11, pp. 4575-4585, Nov. 2008.

[11] P. Almers, K. Haneda, J. Koivunen, V. M. Kolmonen, A. Molisch, A. Richter, J. Salmi, F. Fufvesson, and P. Vainikainen, "A dynamic multilink MIMO measurement system for $5.3 \mathrm{GHz}$," Proc. 29th URSI Gen. Assem., Chicago, USA, Aug. 2008.

[12] L. Ahumada, R. Feick, R. Valenzuela, and C. Morales, "Measurement and characterization of the temporal behavior of fixed wireless links," IEEE Trans. Veh. Techno., vol. 54, no. 6, pp. 1913-1922, Nov. 2005.

[13] L. Jiang, L. Thiele, and V. Jungnickel, "Modeling and measurement of MIMO relay channels," Proc. VTC'08-Spring, Singapore, May 2008, pp. 419-423.

[14] 3GPP TR 25.996, "Spatial channel model for multiple input multiple output (MIMO) simulations (Rel. 6)," Sept. 2003.

[15] P. Kyosti et al., "WINNER II channel models," IST-WINNER II D1.1.2, Nov. 2007.

[16] G. Senarth et al., "Multi-hop relay system evaluation methodology (Channel model and performance metric)," IEEE 802.16j-06/013r3, Feb. 2007

[17] C.-X. Wang, X. Hong, X. Ge, X. Cheng, G. Zhang, and J. Thompson, "Cooperative MIMO channel models: A survey," IEEE Commun. Mag., vol. 48, no. 2, pp. 80-87, Feb. 2010.

[18] C. Oestges, N. Czink, B. Bandemer, P. Castiglione, F. Kaltenberger, and A. Paulraj, "Experimental characterization and modeling of outdoorto-indoor and indoor-to-indoor distributed channels," IEEE Trans. Veh. Techno., vol. 59, no. 3, pp. 2253-2265, June 2010 .

[19] W. Xu, S. A. Zekavat, and H. Tong, "A novel spatially correlated multiuser MIMO channel modeling: Impact of surface roughness," IEEE Trans. Antennas Propag., vol. 57, no. 8, pp. 2429-2438, Aug. 2009.

[20] X. Yin, "Spatial cross-correlation of multilink propagation channels in amplify-and-forward relay systems," Proc. IWonCMM'10, Beijing, China, Aug. 2010, pp. 1-5.

[21] N. Czink, B. Bandermer, G. Vazquez-Vilar, A. Paulraj, and L. Jalloul, "July 2008 radio measurement campaign: Measurement documentation," Tech. Rep., COST 2100 TD(08)620, Oct. 2008.
[22] X. Cheng, C.-X. Wang, D. I. Laurenson, S. Salous, and A. V. Vasilakos, "An adaptive geometry-based stochastic model for non-isotropic MIMO mobile-to-mobile channels," IEEE Trans. Wireless Commun., vol. 8 , no. 9, pp. 4824-4835, Sep. 2009.

[23] X. Cheng, C.-X. Wang, D. I. Laurenson, S. Salous, and A. V. Vasilakos, "New deterministic and stochastic simulation models for non-isotropic scattering mobile-to-mobile Rayleigh fading channels", Wireless Commun. and Mobile Computing, John Wiley \& Sons, vol. 11, no. 7, pp. 829-842, July 2011.

[24] A. Abdi, J. A. Barger, and M. Kaveh, "A parametric model for the distribution of the angle of arrival and the associated correlation function and power spectrum at the mobile station," IEEE Trans. Veh. Techno., vol. 51, no. 3, pp. 425-434, May 2002.

[25] C.-X. Wang, M. Pätzold, and Q. Yao, "Stochastic modeling and simulation of frequency correlated wideband fading channels," IEEE Trans. Veh. Techno., vol. 56, no. 3, pp. 1050-1063, May 2007.

[26] C.-X. Wang, D. Yuan, H.-H. Chen, and W. Xu, "An improved deterministic SoS channel simulator for efficient simulation of multiple uncorrelated Rayleigh fading channels," IEEE Trans. Wireless Commun., vol. 7, no. 9, pp. 3307-3311, Sep. 2008.

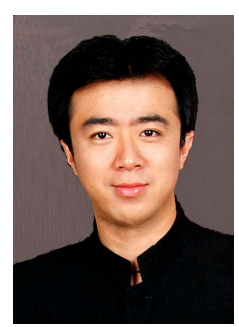

Xiang Cheng (S'05-M'10) received the BSc and MEng degrees in communication and information systems from Shandong University, China, in 2003 and 2006, respectively, and the $\mathrm{PhD}$ degree from Heriot-Watt University and the University of Edinburgh, Edinburgh, UK, in 2009, where he received the Postgraduate Research Thesis Prize.

Dr. Cheng has been a lecturer at Peking University, Beijing, China since 2010. His current research interests include mobile propagation channel modeling and simulation, multiple antenna technologies, mobile-to-mobile communications, and cooperative communications. He has published more than 30 research papers in journals and conference proceedings.

Dr. Cheng was awarded the "2009 Chinese National Award for Outstanding Oversea PhD Students" for his academic excellence and outstanding performance. He served as a Symposium Vice-Chair for CMC 2010 and CMC 2011.

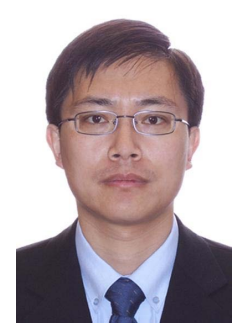

Cheng-Xiang Wang (S'01-M'05-SM'08) received the BSc and MEng degrees in Communication and Information Systems from Shandong University, China, in 1997 and 2000, respectively, and the PhD degree in Wireless Communications from Aalborg University, Denmark, in 2004.

He has been with Heriot-Watt University, Edinburgh, UK, since 2005, first as a Lecturer, then as a Reader in 2009, and then as a Professor since 2011. He was a Research Fellow at the University of Agder, Grimstad, Norway, from 2001-2005, a Visiting Researcher at Siemens AG-Mobile Phones, Munich, Germany, in 2004, and a Research Assistant at Technical University of Hamburg-Harburg, Hamburg, Germany, from 2000-2001. His current research interests include wireless channel modeling and simulation, green communications, cognitive radio networks, vehicular communication networks, mobile femtocell networks, cooperative (relay) MIMO communications, and (beyond) 4G wireless communications. He has published 1 book chapter and more than 150 papers in refereed journals and conference proceedings. Dr Wang is serving as an Associate Editor for IEEE Transactions on Vehicular Technology and served as an Editor for IEEE Transactions on Wireless Communications (2007-2009). $\mathrm{He}$ was the leading Guest Editor for IEEE Journal on Selected Areas in Communications, Special Issue on Vehicular Communications and Networks. He served or is serving as a TPC member, TPC Chair, and General Chair for more than 60 international conferences. He received the IEEE Globecom'10 Best Paper Award in 2010 and the IEEE ICCT'11 Best Paper Awards in 2011. 


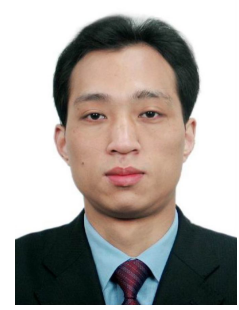

Haiming Wang received the M.S. and Ph.D. degrees in electrical engineering from Southeast University, Nanjing, China, in 2002 and 2009, respectively. He joined the School of Information Science and Engineering, Southeast University, in April 2002. Now he is an associate professor. His current research interests include signal processing for MIMO wireless communications and millimeter-wave wireless communications. Dr. Wang received the first-class Science and Technology Progress Award of Jiangsu Province of China in 2009.

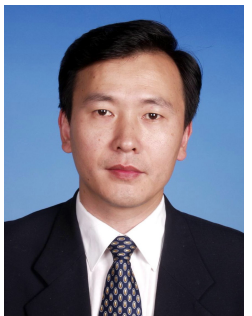

Xiqi Gao (SM'07) received the Ph.D. degree in electrical engineering from Southeast University, Nanjing, China, in 1997. He joined the Department of Radio Engineering, Southeast University, in April 1992. Since May 2001, he has been a professor of information systems and communications. From September 1999 to August 2000, he was a visiting scholar at Massachusetts Institute of Technology, Cambridge, and Boston University, Boston, MA. From August 2007 to July 2008, he visited the Darmstadt University of Technology, Darmstadt, Germany, as a Humboldt scholar. His current research interests include broadband multicarrier communications, MIMO wireless communications, channel estimation and turbo equalization, and multirate signal processing for wireless communications. He serves as an Associate Editor for the IEEE Transactions on Signal Processing and the IEEE Transactions on Wireless Communications.

Dr. Gao received the Science and Technology Progress Awards of the State Education Ministry of China in 1998, 2006 and 2009.

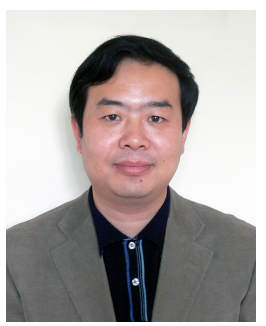

Xiaohu You (SM'11) was born on August 25, 1962. $\mathrm{He}$ received his Master and Ph.D. degrees from Southeast University, Nanjing, China, in Electrical Engineering in 1985 and 1988, respectively. Since 1990, he has been working with National Mobile Communications Research Laboratory at Southeast University, where he held the rank of professor. His research interests include mobile communication systems, signal processing and its applications. He has contributed over 40 IEEE journal papers and 2 books in the areas of adaptive signal processing, neural networks and their applications to communication systems. From 1999 to 2002, he was the Principal Expert of the C3G Project, responsible for organizing China's 3G Mobile Communications R\&D Activities. From 2002 to 2006, he was the Principal Expert of the national 863 Beyond 3G FuTURE Project. Now he is the member of China 863 expert committee responsible for telecommunications.

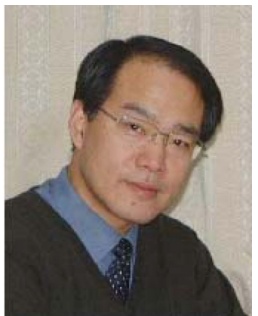

Dongfeng Yuan (SM'01) received the M.S. degree in Department of Electrical Engineering, Shandong University, China, in 1988, and got the Ph.D. degree in Department of Electrical Engineering, Tsinghua University, China in January 2000. Currently he is a full professor and dean in School of Information Science and Engineering, Shandong University, China. From 1993-1994, he was with Electrical and Computer Department at the University of Calgary, Alberta, Canada. He was with Department of Electrical Engineering in the University of Erlangen, Germany from 1998 to 1999; with Department of Electrical Engineering and Computer Science in the University of Michigan, Ann Arbor, USA, from 2001 to 2002; with Department of Electrical Engineering in Munich University of Technology, Germany in 2005, and with Department of Electrical Engineering Heriot-Watt University, UK, in 2006. His current research interests include cognitive radio systems, cooperative (relay) communications, and $4 \mathrm{G}$ wireless communications.

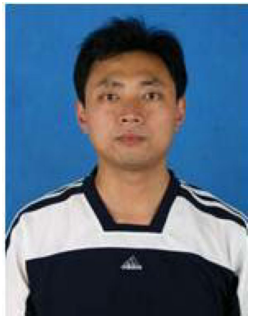

Bo Ai (M'00-SM'09) is now working in Beijing Jiaotong University as a professor and Ph.D. Supervisor. He is vice director of State Key Lab. of Rail Traffic Control and Safety. He has authored 5 books and published over 124 scientific research papers in his research area. He has hold 5 national invention patents and 1 US patent. He has been the research team leader for 13 national projects. He has won some scientific research prizes, such as the First Grade of Technology Advancement Award of Shanxi Province. He is an IEEE senior member and serving as the editorial member of IEEE Transactions on Consumer Electronics and Wireless Personal Communications, a publication editor for Ubiquitous Computing and Communication Journal. His research interests include radio wave propagation and wireless channel modeling, power amplifier pre-distortion, LTE-R system and Cyber-physical System.

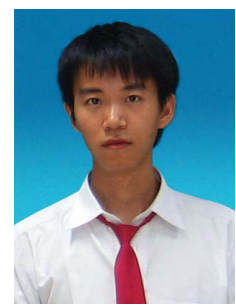

Qiang Huo received the B.S. degree in electronic and information engineering from South China University of Technology, China in 2009. Since September 2009, he has been a Ph.D. candidate in Peking University, China. His main research interests include adaptive signal processing, estimation and optimization theory, cooperative communications, physical network coding and wireless propagation channel modeling and simulation.

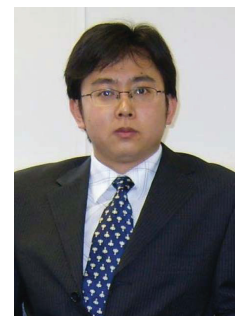

Ling-Yang Song (S'03-M'06) received a $\mathrm{PhD}$ from the University of York, UK, in 2007, where he received the K. M. Stott Prize for excellent research. He worked as a postdoctoral research fellow at the University of Oslo, Norway, until rejoining Philips Research UK in March 2008. Now, he is a full professor with School of Electronics Engineering and Computer Science, Peking University, China. His main research interests include adaptive signal processing, cognitive and collaborative communications. He is co-inventor of a number of patents and author or co-author of over 100 journal and conference papers. $\mathrm{He}$ is currently on the Editorial Board four International Journals, and serves as a member of Technical Program Committee and Co-chair for several international conferences and workshops. He is a member of the IEEE and IEEE ComSoc.

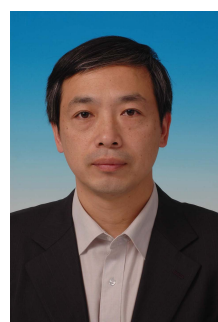

Bing-Li Jiao received B.S. and M.S. degree from Peking University, China in 1983 and 1988, respectively, and received Ph.D. from University of Sarrbruecken, F. R., Germany in 1995. Then, he became an associate professor and professor with Peking University in 1995 and 2000, respectively. His current interests include communication theory and techniques and sensor design. 